Borbála Rózsa Zsindely

\title{
Zusätze zur Geschichte der kleinen Mühlen des Komitat Baranya in 20. Jahrhundert $^{1}$
}

\begin{abstract}
In the light of the milling industry, which flourished nationwide at the turn of the century, the study deals with Baranya County's position, primarily examining the possibilities and limitations of operating small mills in the region later. The author outlines this sector's prosperity before 1945 based on the family archives of the owner of the contemporary journal, „Molnárok Lapja” and the Orfü mills. The sources suggest that the mill industry in Baranya cooperated in an organized manner, both at the county and at the smaller, local levels. For example, the relationship between the mills and the Danube Steamship Company (DGT) was established through forced local cooperation. This concentration is to be interpreted as a local specificity, as it was in DGT's interest to know the level of watercourses due to mining. Due to coal mining, the water levels of the local streams dropped, which made it very difficult for small mills to operate. On the other hand, the mills also needed coal, as did the Serbs who temporarily occupied the region after the First World War, who also removed the extracted coal from the area. After the years of consolidation, the first sensitive blow to the small mills in Baranya was the economic crisis, when as a result of the quota regulation, they were forced to limit their production significantly and were obliged to supply the milled grain. Their fate was sealed together by the post-1945 nationalization decrees and the associated withdrawal of water use rights.
\end{abstract}

Keywords: mill industry history, small mill industry, overshot mill wheel, millers' association, Molnárok Lapja

\section{Einführung}

Ein wichtiger Aspekt der Geschichte der ungarischen Mühlenindustrie, der allerdings wenig bekannt ist, ist die Geschichte der Kleinmühlenindustrie. Die ungarische Kleinmühlenindustrie, die in erster Linie am Land tätig war, war der lokalen und regionalen Nachfrage angepasst und versorgte die Bevölkerung mit Mehl, Schießpulver und mit Fries. Ich möchte in erster Linie einen Teil der weitvermahlenden Kleinmühlenindustrie in meiner Arbeit vorstellen. Auf den folgenden Seiten untersuche ich die Geschichte der Kleinmühlenindustrie im Komitat Baranya mit dem Ziel, die lokale Bedeutung der Kleinmühlenindustrie diesen Komitats in der Zeit der Jahrhundertwende bekannt zu machen. Am Anfang meiner Arbeit stelle ich die Grundlagen der Mühlenindustrie Ungarns und in der Zeit vor. Damit im Zusammenhang erläutere ich die verschiedenen historischen Ereignisse, den Einfluss auf die Entwicklung der ungarischen Mühlerindustrie ausgeübt haben. Danach stelle ich die schemenhafte Geschichte der Kleinmühlen im Komitat Baranya vor. Dazu ist meine wichtigste Quelle die Molnárok Lapja $^{2}$ die von 1904 bis 1938 die wichtigste und bedeutendste ungarische Fachzeitung für die Mühlenindustrie war. Sie bot ein fachliches Forum und war ein Kommunikationskanal.In meiner Arbeit stelle ich die Texte aus der Molnárok Lapja vor, den Einfluss auf die Mühlenindustrie des Bezirks ausübten. Am Ende meiner Arbeit beschreibe ich die Kleinmühlenindustrie der Stadt Fünfkirchen (Pécs) in der Abfolge wie diese Mühlen an den städtischen Bächen auffindbar waren. Für diesen Teil war meine wichtigste Quelle die Projektbeschreibung von György Bálints

Ich widme meine Arbeit der Erinnerung an meine Großmutter, Magda Mészöly!

auf Deutsch: Müllerzeitung 
Mühlenforschungsprojekt ${ }^{3}$, die ich von Kata Füzes, Leiterin der Mühlen in Orfü, bekommen habe. Am Ende fasse ich die Ergebnisse meiner Forschung kurz zusammen und ziehe eine Schlussfolgerung.

\section{Was man über die Mühlenindustrie in der Epoche wissen soll}

Nach dem Ausgleich (1867) entwickelte sich die ungarische Mühlenindustrie schnell. Neue Frästechniken wurden erprobt und die wissenschaftliche Forschungen der Lebensmittelchemie waren erfolgreich. Die größten Wissenschaftler der Epoche (Tamás Kosutány ${ }^{4}$, Hankóczy Jenó $^{5}$, Grutzl Ferenc ${ }^{6}$ ) experimentierten damit, die idealen Frästechniken zu finden und deren wissenschaftlichen Ereignisse und die Qualität des ungarischen Getreides zu dokumentieren und Eigenschaften zu definieren. Tamás Kosutány untersuchte erstmals das ungarische Getreide chemisch, er erkannte, dass das ungarische Getreide einen größeren Glutenanteil enthält, als ausländisches Getreide. Natürlich darf die Maschinenfabrik von Abraham Ganz nicht vergessen werden, genauso wenig wie die in seiner Fabrik arbeitenden Gebrüder Haggenmacher. Károly Haggenmacher stellte das erste Flachsieb her. Damit konnten schon die Graupen sortiert werden und die Qualität-des vermahlenden Getriedes wesentlich verbessert werden. ${ }^{7}$

Hinter dem Erfolg der ungarischen Mühlenindustrie müssen aber mehr Faktoren gesucht werden. Die fachliche Überlegenheit und Bewusstheit half dabei, die Menge und die Qualität des Getreides zu verbessern. Ungarn stand auf dem zweiten Platz der Mehlexportweltrangliste, einzig die Vereinigten Staaten exportierten mehr. Riesengroße Mengen von Mehl wurden mit Schiffen über Fiume oder über die Donau Richtung Wien aus dem Land geliefert. Unter den Zielländern fanden sich selbst solche fernen Länder wie Chile, Peru und Indonesien.

Die größeren Dampfmühlen wurden in der Hauptstadt (Budapest) in der zweiten Hälfte des 18. Jahrhunderts gebaut. An die Donau geliefertes Getreide wurde hier gemahlen und weitertransportiert. Deshalb wurde Budapest zum mühlenindustriellen Zentum, weil die Handelswege aus dem Balkan, Transilvanien, aus dem Norden und aus Wien sich hier trafen und weil die nötige logistische Infrastruktur hier zur Verfügung stand. Der Verkehr wurde viel schneller und moderner, deshalb konnten die Lieferzeiten gesenkt werden und die ungarischen Getreideproduzenten konnten an den Weltmarkt liefern.

\section{Lokale Ereignisse, die auf die Mühlenindustrie Einfluss ausübten}

Die Blütezeit der Mühlenindustrie fängt in Ungarn im ersten Drittel des 19. Jahrhunderts mit der Dampfmühlengründungsenwelle an. „Unser Müblenindustrie war schon in den 186070-er Jabren im Weltmaßstab führend. "8 Nach der Krise von 1873 wurden weniger Mühlen

\footnotetext{
3 Balázs, György: Malmok Baranyában.(Handschrift) Szentendre, 2015. Entstanden mit der Titel „Kutatás und Studien Herstellung „Miller's Route“ gekennzeichnet mit der Nummer HUHR/1101/1.2.3/0012. Ich bedanke mich bei Kata Füzes, die Besitzerin der Orfüer Mühlen, dass sie ihr Familienarchiv zu meiner Verfügung gestellt hat. Das Archiv wurde zu einer wichtigen Quelle meiner Arbeit.

4 Pintér, János: Kosutány Tamás. In: https://mek.oszk.hu/00000/00058/html/kosutany.htm (letztes mal herunterladen am: 04. 02. 2020.)

5 Ders.

6 P. Hartyányi, Borbála: Hankóczy Jenő. In: https://mek.oszk.hu/00000/00058/html/kosutany.htm (letztes mal herunterladen am: 04. 02. 2020.)

7 Zsindely, Borbála Rózsa: Egy korabeli szaklap és annak szerepe a biatorbágyi Ilona-hengermümalom történeti kutatásában. Diplomarbeit, Universität Pécs, Philosophische Fakultät, Ungeteilte Lehrerbildung, Themenbereuerin: Frau Dr. habil. Virág Rab Phd. Jahr der Schützung: 2020.

8 Katus, László: A tőkés gazdasági fejlődés a kiegyezés után.In: Magyarország története 1848-1890.2. Hergestellt von Endre Kovács.1007. Zitiert noch: Klement, Judit: A hazai vállalkozók a hőskorban. A budapesti gőzmalomipar
} 
gebaut, aber die Dampfmühlengründungswelle blieb nicht stehen, die Entwicklung wurde nur langsamer. Aber die Frage, ob die Österreichisch-Ungarische Monarchie funktionierte, lag nicht an der wirtschaftlichen Entwicklung. ${ }^{9}$ Die Entstehung des ungarischen Kapitalismus und deren Blütezeit steht im Zusammenhang mit dem „mit wirklicher Spitzentechnologie arbeitenden ersten ungarischen Schlüsselsektor"10 der Mühlenindustrie. Budapest wurde nach Minneapolis das weltweite zweite Zentrum der Mühlenindustrie. Die ungarische Großmühlenindustrie war auf Mehlexport ausgerichtet, während die Kleinmühlenindustrie den lokalen und regionalen Bedarfbefriedigten. Wegen des traditionellen Gesellschaftsmodells konnte die kapitalistische Umwandlung nicht im ganzen Land durchgeführt werden, die Rudimente der feudalistischen Ordnung waren noch am Anfang des 20. Jahrhunderts sichtbar. Das ins Land einfließende österreichische Kapital wurde für die Verwirklichung der kapitalistischen Entwicklungen gebraucht, weil das im Inland sich angesammelte Kapital für die Finanzierung der Modernisierungen nicht ausreichend war. Nach dem Ausbruch des Ersten Weltkrieges musste die Mühlenindustrie sich an andere wirtschaftliche Verhältnisse anpassen. Eine Erscheinung davon war die Einführung der neuen Mahlordnung (Es wurde verboten feines Mehl zu produzieren, stattdessen sollte das Mehl mit Ersatzmaterialien gemischt werden). Daneben wurde es auch schwieriger den Rohstoff einzuholen ${ }^{11}$ und zu den Mühlen zu liefern. Nach Trianon erlitt die Mühlenindustrie einen großen Verlust. Die Rohstoffversorgung sichernde Regionen (Batschka und Banat) wurden abgetrennt, was die Arbeit der Großmühlenindustrie, die exportierte, unmöglich machte. Die Budapester Großmühlen versuchten eine Zeitlang Getreide aus den Nachbarländern zu verarbeiten, aber sie mussten wegen fehlenden Geldes und Rohstoffe die Produktion einstellen. Wenn die ungarischen Kleinmühlen aus ihrem Umkreis genügend Rohstoff beziehen konnten, dann konnten diese Mühlen innerhalb der neuen Grenzen Ungarns weiter mahlen. Natürlich gab es viele Mühlen, die nach dem Weltkrieg und nach dem Frieden der existentiellen unklaren Lage zum Opfer fielen. Die große Wirtschaftskrise verschonte auch die Mühlenindustrie nicht. Die Mühlen, die die Krise überlebten, wurden im Zweiten Weltkrieg (Bombenschäden, Explosionen, Brände usw.) oder von dem nachher kommenden neuen Staatsapparat und dessen schrittweise erlassenen Entprivatisierungserlässen $(1948,1952)$ ruiniert.

Der Grund des bis heute spürbaren Verlustes für die ungarische Provinz ist der hier skizzierte Ablauf. Das folgende Diagramm stellt das 100 Jahre dauernde Schrumpfen der Mühlenindustrie dar, die Daten stammen von Tibor Molnárf. ${ }^{12}$ Das Diagramm stellt eindeutig den größten Rückgang der Mühlenindustrie dar. Der größte Einbruch in der Produktion der Mühlen waren die drei Jahrzehnte nach der Jahrhundertwende. Die Jahre, aus denen die Daten stammen, sind die Jahren, in denen das Statistische Amt Messungen durchführte. Die Häufigkeit der Messungen sind nicht immer gleich. Das Diagramm zeigt anschaulich, wie die Zahl der Mühlen sinkt. Heute ist die Zahl der noch funktionierenden oder als Museum betriebenen Mühlen circa hundert.

\footnotetext{
vállalkozói a 19. század második felében. Budapest, 2012.

9 Klement, Judit: A hazai vállalkozók... 20.

10 Ders. 8.

11 Ders. 10.

12 Molnárfi, Tibor: A malomipar történeti-statisztikai feldolgozásának elméleti és gyakorlati kérdései. Történeti statisztikai évkönyv 1967 - 1968. Központi Statisztikai Hivatal Könyvtár, Országos Levéltár. 291 - 346.
} 
Figur 1: Zahl der Mühlen.

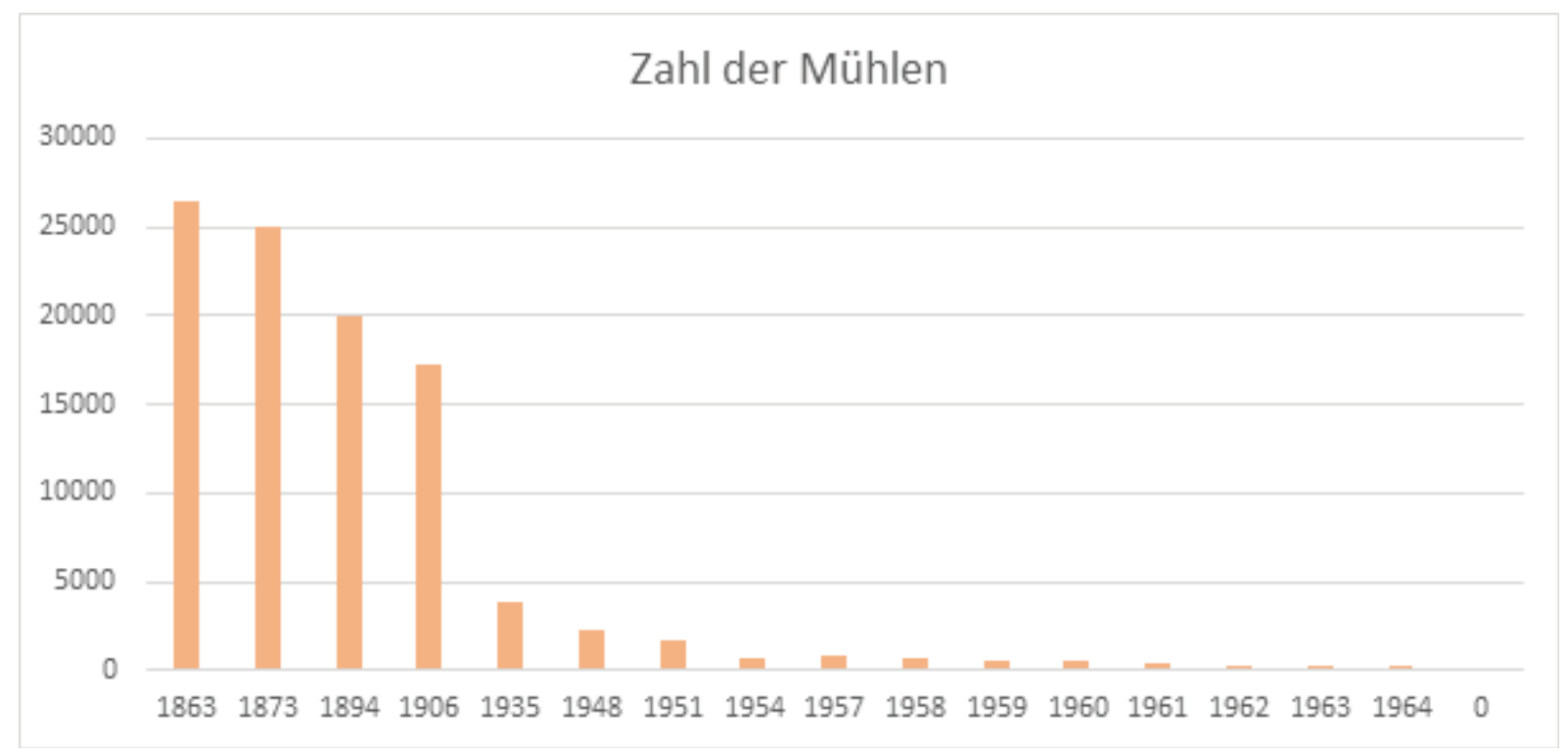

Quelle: Molnárf, Tibor: A malomipar... 291 - 346.

\section{Situation der Mühlen im Komitat Baranya}

Die Mühlen aus dem Komitat Baranya wurden am Anfang des 19. Jahrhunderts steuerlich erfasst. Im Jahre 1815 waren 349 Mühlen in 212 Siedlungen tätig. Bis 1841/42 sank die Zahl der Mühlen auf 207. Die Mühlen zu dieser Zeit hatten oberschlächtige Wasserräder und funktionierten mit ein oder zwei Rädern. Die Mühlen konnten nach Regen oder nach der Schneeschmelze mahlen. Viele gerichtliche Auseinandersetzungen sorgten dafür, dass die Ausstattung vieler Mühlen dokumentiert ist. Streit zwischen Mühlen und Siedlungen um Wasserrechte führte zu diesen Prozessen. ${ }^{13}$ Ein weiterer Grund waren Konflikte um Besitzund Erbverhältnisse. Konsolidierte sich die wirtschaftliche Lage entstanden schnell neue Mühlen an den Ufern der Bäche, um den lokalen Bedarf zu sichern. Schon seit den 1730-er Jahren verlangten die Grundherren für die Wassernutzung Steuern. Auch für die Nutzung der Arbeits- und Wohnhäuser verlangten die Grundherren eine Abgabe. Die Müller erfüllten meist vorbehaltlos die Konditionen des Grundherrens. Im Szentlőrincer járás ${ }^{14}$ und im Siklósi járás ${ }^{15}$ gab es aus Holz gebaute Mühlen, die einfach eingerichtet waren. Wir können aber trotzdem von gut ausgestatteten Mühlen sprechen. Im Mohácsi járás ${ }^{16}$ um Pécsvárad war die Leistung der Mühlen kleiner und sie konnten nur die lokale Nachfrage sättigen. In Mohács arbeiteten die Mühlen mit Wasserkraft. Die Besitzer der Schiffs-und Trockenmühlen um Mohács waren hier noch keine offiziellen Handwerker. An Donau und an Drau mahlten 54 Schiffsmühlen und hier waren die Müller bereits Handwerker. Um diese Mühlen miteinander zu vergleichen schlägt Mária Móró in ihrem Aufsatz vor, Gerichts-und Kämmereiunterlagen exakt zu untersuchen. ${ }^{17}$

Für den untersuchten Zeitraum erschienen 1904 die ersten erwähnenswerten Berichte aus dem Komitat in der Müllerzeitung. In diesem Jahr wurde die Müllerzeitung auch gegründet. Im zweiten Teil der Zeitung finden sich zwei interessante Ereignisse. Das Erste war, dass die Bevölkerung als Wunder erwähnt, dass das aus frisch gemahlenem Mehl gebackene Brot sich

13 Móró, Mária Anna: Malmok Baranya megyében a 18. században. In: Dél-Dunántúl népi építészete. Hergestellt von: L. Imre, Mária- Cseri, Miklós. Szentendre - Pécs, 1991.

14 auf Deutsch: St. Laurenzer Kreis

15 auf Deutsch: Siegloser Kreis

16 auf Deutsch: Mohatscher Kreis

17 Móró, Mária Anna: Malmok Baranya megyében a 18. században... 
verhärtete. Später kam ans Tageslicht, dass das Mehl nicht ganz rein war, weshalb das Brot versteinerte. Das andere Ereignis war eine Beschreibung einer tragbaren Mühle, die eine große technische Neuerung war. ${ }^{18}$ Auch im diesem Jahr kam es zu mehreren Personalwechseln (Mühlen haben Besitzer gewechselt), was auch beweist, dass im Personal der Mühlen Änderungen vorkamen. ${ }^{19}$ Ein weiteres hervorzuhebendes Ereignis dieses Jahres ist, dass die Mühlenbesitzer aus dem Komitat Baranya eine Eingabe an den Justizminister Sándor Plósz ${ }^{20}$ schieckten, um die Streichung der Wassermieten zu erreichen.

Der Grund der Eingabe war, dass das Wasser der Bäche von den Bauern für die Bewässerung verwendet wurde. Oft blieb kein Wasser im Bachbett, womit die Mühlen betrieben werden konnten. ${ }^{21}$ Aus dem Jahr 1905 findet sich der interessanteste Artikel der Müllerzeitung in der Nummer 23. In dem Artikel können wir darüber lesen, dass sie Mühlenbesitzer aus Baranya sich entschieden haben in allen großen Städten die Funktionsweise von Mühlen zu unterrichten. Damit konnte das Problem der schlechten Ausbildung gelöst werden. Aus diesem Jahr gibt es mehrere Berichte, die auf logistische Fragen, die zwischen Mühlen entstanden verweisen: zum Beispiel wie stark die Mühlsteine sind. ${ }^{22}$ Im Jahre 1906 kann man in der Müllerzeitung über die Mühlen aus Baranya über Personalwechsel ${ }^{23}$, Mühlen-Umbauten ${ }^{24}$ und Besitzerwechsel lesen. ${ }^{25} \mathrm{Im}$ Jahre 1907 wechselten für längere oder kürzere Zeit mehrere Mühlen-Besitzer. ${ }^{26}$ Daneben experimentierten einige Mühlen mit der Maisproduktion. Der Mais wurde oft von österreichischen Handelsvertretern verkauft. ${ }^{27}$ Im Jahre 1908 konnten die meisten Mühlen aus Branau wegen dem harten Winterfrost nicht mahlen, da die Bäche eingefroren waren. Eine Ausnahme bildeten Wassermühlen, die einen eigenen Motor oder Dampfmaschine hatten. In der Zeitung wurden die Mühlenbesitzer vor dem kalten Winter gewarnt und ihnen wurde geraten - wenn möglich - eine solche Maschine anzuschaffen. ${ }^{28}$ In diesem Jahr begannen die Mühlerbesitzer aus dem Komitat Baranya unter dem Namen Mehlverkaufsgenossenschaft der Mühlen aus dem Komitat Baranya zu kooperieren. Am 25. Juli: 1908 war in Fünfkirchen, in einem Privatzimmer des König Matthias Hotel, eine vorbereitende Kommissionssitzung geplant, zu der auch der Chefredakteur der Müllerzeitung, Ödön Jutassy, oder seine Vertreter eingeladen waren. Hintergrund der Genossenschaft war, dass gedacht wurde, dass Kleinmühlen alleine schwer ihr Glück im Bereich des Mehlverkaufs finden. Für den Verkauf sind Transport, Getreide und Beziehungen nötig. Deshalb dachten die Mühlenbesitzer, dass sie in einer Genossenschaft zusammen mehr Möglichkeiten haben können. ${ }^{29}$

Auch im Jahre 1921 kamen die Mühlenbesitzer aus dem Komitat Baranya in der Fachzeitung vor. Genauso wie in den Jahren 1917 und 1918, in denen es aber nur um den Verkauf und Vermiete einer Mühle ging. ${ }^{30}$ Zwischen 1918 und 1921 stand mehr als die Hälfte des Komitats unter serbischer Besetzung, die Mühlenindustrie des Komitats befand sich in einer wirklich

\footnotetext{
18 Molnárok Lapja, 2. Nr. 09. 01.1904.27.

19 Ders. 11. (1914) Nr. 22-23. 476.

21 Molnárok Lapja, 11. (1914) Nr. 24.520.

22 Ders. 12. (1915) Nr. 20. 30. 05.443.

23 Ders. 13. (1906) Nr. 7.17.02.160. und Nr. 9. 10. 03. 232.

24 Ders. 13. (1906) Nr. 28. 14.07.675.

5 Ders. 13. (1906) Nr. 45. 10.11.1088.

26 Ders. 14. (1907) Nr. 4. 26.01. 83. und Nr. 19. 11.05. 502.

27 Ders. 14. (1907) Nr. 50. 14. 12. 1258. und Nr. 51. 21.12.1313.

28 Ders. 15. (1908) Nr. 7.15.02.158.

29 Ders. 15.(1908) Nr. 19. 18.07.714.

30 Ders. 14. (1917) Nr. 38. 21. 09. 792. und Nr. 45. 10.11. 930.
}

20 Sándor Plósz (1846 - 1925) war Justizminister zwischen 26. 02.1899 und 18. 06 1905, Jurist, Universitätslehrer, Mitglied der Széll-, Khuen-Héderváry und die ersten István Tisza Regime, als unabhängige. Sein Nachfolger war Bertalan Lanyi bis 21. 04. 1906, aus der Szabadelvü Partei Ungarische Lebenslauf Lexikon http://mek.oszk. hu/00300/00355/html/ABC11587/12280.htm (letztes mal herunterladen am 01.11.2020.) 
schweren Situation. Viele Mühlen mussten die Produktion einstellen, da sie dauernd mit Rohstoff- und Kohlemangel kämpfen mussten. Das gemahlene Getreide beanspruchten die Serben und nicht nur das Mehl, sondern auch die Arbeit wurde nicht bezahlt. ${ }^{31}$ Im August 1921 zogen die Serben aus der Region ab und die Mühlenindustrie des Komitats konnte von Neuem starten. Im September 1921 forderten die Mühlenbesitzer des Komitats bei der Ländlichen Müllergenossenschaft schriftlich, dass der freie Verkehr so früh wie möglich wieder einzuführen ist und die Kosten für Kohle in Fünfkirchen gesenkt werden. Ihrer Bitte folgte das Versprechen der Regierung die Maßnahmen in kurzer Zeit umzusetzen. ${ }^{32} \mathrm{Zu}$ diesem Zeitpunkt ist bereits die Quoten-Regelung mit der Nummer 1921/XXXIX. ${ }^{33}$ gültig. Der wichtigste Abschnitt der Quoten-Regelung, die auch die Mühlenindustrie des Komitats Branau betraf, ist die Abschaffung des freien Mahlverkehrs zu Gunsten des gebundenen Mahlverkehrs. Damit wollte die Regierung erreichen, dass ein Teil des Getreide abgeliefert wird, damit Menschen, die keine Nahrung haben, Nahrung bekommen und dass das Schwarzmahlen und der Schmuggel von Getreide eingegrenzt wird. Das langfristige Ziel war, das Land nach schweren wirtschaftlichen, gesellschaftlichen und politischen Krisen neu wiederherzustellen. Der größte Nachteil des gebundenen Mahlverkehrs war, dass die Produzenten aus einer sehr schwerer Position kamen. Die Regelung verursachte wegen der ganz schlechten wirtschaftlichen Verhältnisse für viele Mühlen den Untergang. Die Regulierung der Mühlen durch die Quoten-Regelung stockte, weil nicht genug gesät wurde und die Mühlen konnten das Getreide nicht andauernd mahlen, da es nicht rechtzeitig geliefert wurde. Zusätzlich konnten die Mühlen auch nicht auf eigene Rechnung mahlen, da man nicht wissen konnte, wann das Getreide der Quoten-Regelung kam, das in einer bestimmten Zeitspanne gemahlen werden musste. ${ }^{34}$

\section{Kleinmühlen in Fünfkirchen}

Die meisten in diesem Teil genutzten Quellen bekam ich von Kata Füzes, der Leiterin des Orfüer Mühlenmuseums. Dafür bin ich ihr sehr dankbar.

Bei der Betrachtung der Fünfkirchner Kleinmühlen muss man am Anfang unbedingt hervorheben, dass die lokale Mühlenindustrie am Ende des 19. Jahrhunderts und am Anfang des 20. Jahrhunderts eine solche Epoche erlebte, in der die traditionellen - meist Wassermühlen - modernisiert wurden und zu Waltzwerkmühlen umgebaut wurden. Natürlich war dieser Modernisierungsprozess teuer, nicht alle Mühlen konnten ihn sich leisten. Die Mühlen, die sich die Modernisierung nicht leisten konnten, verloren den Anschluss. „Wasserbücher aus dem Ende des 19. und Anfang des 20. Jabrhunderts dokumentieren im südöstlichen Bereich Fünfkirchens Müblen, die mit zwei Müblsteinpaaren arbeiteten und aus einem Doppelweitzenwerk (wahrscheinlich Ganz-Produkt) bestanden." ${ }^{35} \mathrm{Ab}$ dem Jahr 1885 war die Wassernutzung an Genehmigungen gebunden. Die Genehmigung wurde von dem Vizegespansbüro erteilt und später von dem Kulturingenieursbüro. Das letztere verfügt über Beschreibungen über den Ausbau der Wasserwerke, Wasserräder, Bachbette und Schleusen. Wenn eine Mühle mindestens zwanzig Jahre produziert hatte, dann musste sie mit der Bestätigung der Gemeinde keine Gebühren mehr zahlen und die Lizenz war nicht mehr an eine Zeit gebunden.

\footnotetext{
31 Kaposi, Zoltán: Pécs gazdasági helyzete a szerb megszállás idején (1918-1921) Pécsi Szemle, 2011. Frühling

32 Molnárok Lapja, 21. (1921) Nr. 36. 03.09.1.

33 1921./XXXIX. törvénycikk. az ellátatlanok ellátásának biztosítására szolgáló őrlési és forgalmi adóról. In: https://net.jogtar.hu/ezer-ev-torveny?docid=92100039.TVI\&searchUrl=/ezer-ev-torvenyei\%3Fkeyword\%3D\%2 5C5\%2591r1\%25C3\%25A9si\%2520forgalom (letztes mal herunterladen am: 01.11.2020.)

34 1921. évi XXXIX. tv. indoklása az ellátatlanok ellátásának biztosítására szolgáló ôrlési és forgalmi adóról. Von selben Ort 133-188.(letztes mal herunterladen am: 19.10.2020.)

35 Balázs, György: Malmok Baranyában... 25.
} 
Die Mühlenbesitzer und die Ingenieuren mussten gemeinsam die oben erwähnten Eingriffe in die Landschaft planen. Die Planungen wurden in vier Exemplaren ausgefertigt: ein Exemplar für den Vizegespan, eines für das Kulturingenieursbüro, eines für das Kreisarchiv und eines für den Mühlenbesitzer selbst. Die Dokumente vom Ende des 19. Jahrhunderts geben in allen Fällen eine detaillierte Beschreibungen des Wasserrads, des Schleusensystems, der Fallbedingungen des Bachbettes, der Lage von Brücken, der an das Wasserrad gebundenen Teile und Maschinen zum Beispiel Mahlwerk und Sieb und der für den Antrieb gebrauchten Energie. Eventuelle Ausbauten und Veränderungen mussten die Mühlenbesitzer bei dem Vizegespansbüro melden. Die meisten Mühlen behielten den Namen des ersten Besitzers. Ein eventueller Wechsel der Wasserräder musste auch gemeldet werden. Zum Schluss: Allen nach der Verstaatlichung stillstehenden Fünfkirchner Mühlen wurden 1967 alle Genehmigungen entzogen. ${ }^{36}$

Im Folgenden stelle ich schemenhaft die Geschichte der Fünfkirchner Mühlen dar. In Fünfkirchen finden wir in der untersuchten Epoche an vier Wasserläufen Kleinmühlen, deren Namen sind: Szabolcsi-vízfolyás ${ }^{37}$, Ürögi-vízfolyás ${ }^{38}$, Pécs-Meszesi-vízfolyás ${ }^{39}$ und der Tettyepatak $^{40}$. Spätestens bei der Verstaatlichung stellten alle Mühlen ihre Produktion ein.

József Nürnberger und seine Frau Mária Loschert bekamen 1901 die Genehmigung zur Errichtung einer Mühle am Pécser-Wasserlauf. Mit einem oberschlächtigen Wasserrad, mit 8,7 Meter Durchmesser, wurde ein ein Meter breiter Mühlstein, eine Doppelwalze und ein Schleifmittel angetrieben. Die Doppelwalze beinhaltete ein Grieße und ein Feiner. Die Mühle hörte 1929 auf zu mahlen, weil ein Unwetter den Staudamm beschädigte. Das Mühlrad wurde entfernt und der Kanal aufgefüllt. ${ }^{41}$

Auch an diesem Wasserlauf stand die gemeinsame Mühle von Ármin Weisz und György Fulcz. Im Jahre 1909 bekamen sie die Genehmigung für ein oberschlächtiges Wasserrad mit sechs Meter Durchmesser. Die Versorgung mit Wasser war schwierig, da wegen der Nähe des Bergwerks das Wasser von den Bächen weggeleitet wurde. Die Besitzer übertrugen das Wasserrecht an die Erste Donaudampfschiff-Gesellschaft, ${ }^{42}$ wodurch den Bergwerken erlaubt wurde das Wasser zu benutzen. Die Mühle war damit keine Wassermühle mehr. ${ }^{43}$

Verw. Frau János Steiner bekam 1909 am Pécser-Wasserlauf die Betriebsgenehmigung für eine Mühle-mit einem oberschlächtigen Wasserrad mit 4,4 Meter Durchmesser. Das Wassernutzungsrecht hielt 1920 Ádám Schweitzer und seine Frau. Dieses Recht wurde im Jahre 1948 entzogen, aber zu diesem Zeitpunkt produzierte die Mühle schon nicht mehr, der Besitzer nutzte das Wasser nicht mehr. ${ }^{44}$

Der Mühle von Mihály Türr am Szabolcser-Wasserlauf, später eine Dampfmühle, wurde 1894 mit der Arbeit am Maschinenhaus begonnen. ${ }^{45}$ Die Besitzer der zwei Nachbarmühlen waren verw. Frau Steiner János und József Papp. Die Mühle hatte ein oberschlächtiges Wasserrad mit 4,68 Meter Durchmesser, das bis 1901 einen im Durchmesser ein Meter breiten Mühlstein und eine Doppelwalze antrieb. Nach 1901 wurde eine neue Dampfmühle mit einer Maschine von 50 Pferdestärken gebaut. Der Sohn von Mihály Türr und seine Frau baten im Jahre 1923 darum, dass die Stadt das im Jahre 1912 erteilte Wassernutzungsrecht zurücknimmt. 1948

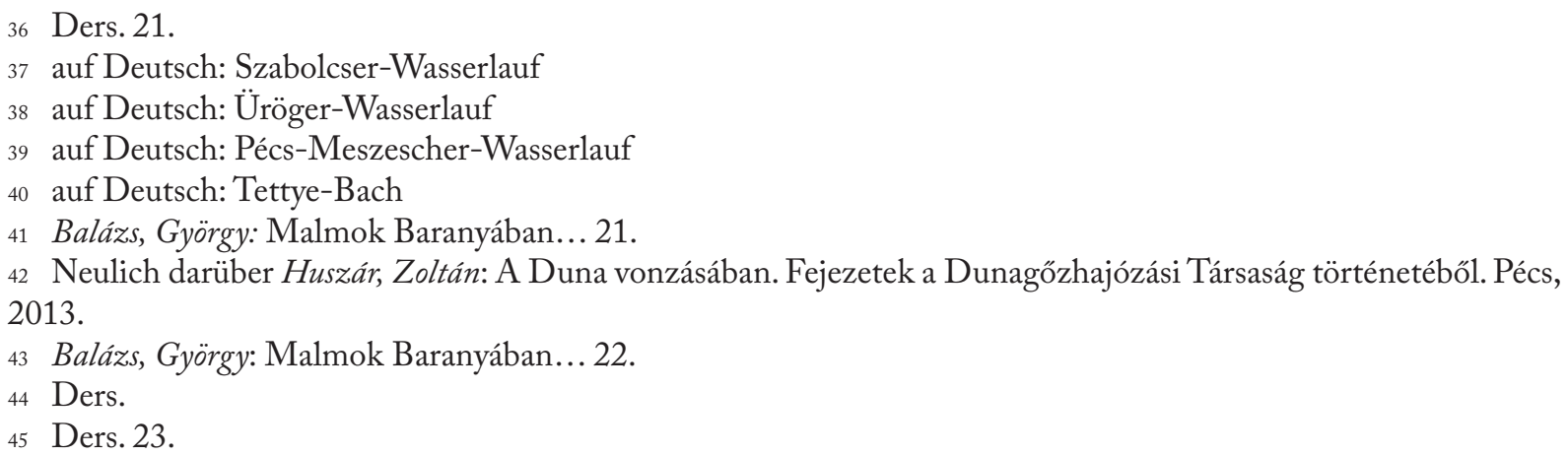


wurde dies vom Bürgermeister bestätigt, allerdings produzierte damals die Mühle schon seit Jahrzehnten nicht mehr. Wegen Veränderungen der Landschaft erreichte kein Wasser mehr die Mühle. ${ }^{46}$

Die Mühle von József Papp wurde bereits erwähnt, sie bekam im Jahre 1912 die Genehmigung. Die Mühle wurde von zwei oberschlächtigen Wasserrädern mit 3,76 Meter Durchmesser angetrieben. Schon 1912 suchte István Papp darum an, dass er die Mühle unter seinem Name führen könne, statt unter dem Namen seines Vaters József Papp. Die Mühle bekam Isván Papp von seinem Vater József Papp als Geschenk. Nach einem-aus dem Jahre 1914 stammenden Protokoll hatte die Mühle die Erlaubnis für zwei Räder, aber nur eines funktionierte auch wirklich. ${ }^{47}$

Der Bestizer der Basa-Mühle war János Piribauer, der eine Genehmigung für den PécserWasserlauf bekam. Er suchte 1901 um die Genehmigung an und bekam sie 1909. Seine Mühle hatte ein oberschlächtiges Wasserrad, das einen Durchmesser von 5,68 Meter hatte und einen Mühlstein mit 0,98 Meter Durchmesser und eine Doppelwaltze antrieb. Die Mühle war noch im Jahre 1912 im Besitz von János Piribauer, seines Sohnes Jenö, die Genehmigung bekamen sie für unbestimmte Zeit. Die Mühle wurde von der Donaudampfschiff-Gesellschaft bei einer Auktion von Gusztáv Graumann mit Einschränkungen unter Bedingungen gekauft. Die Mühle produzierte bis 1942, die Wassernutzungsrechte nahm die Stadt zurück. ${ }^{48}$

Südlich der Stadt zwischen den Megyei und Füzes Gewannen stand die Megyei-Mühle, aber laut der technischen Zeichnung von Ignátz Lukrits hatte der Baugrund keine Parzellenzahl.

Im Racstadtteil standen ab dem Ende des 18. Jahrhunderts zwei Mühlen, die dem Jesuitenorden gehörten. Beide hatten oberschlächtige Wasserräder, eine Mühle hatte ein Rad und die andere zwei. Grundsätzlich wissen wir über die Planungen nur aus Archivmaterialien. ${ }^{49}$ An dem Üröger-Wasserlauf stand eine Wassermühle, sie gehörte 1908 János Inhoff und János Rakk. Im Jahre 1910 bekamen sie das Wassernutzrecht. Die Mühle hatte ein oberschlächtiges Wasserrad, mit 5 Meter Durchmesser. Ein Jahr später wurde die Mühle als die Mühle von János Inhoff erwähnt, zu diesem Zeitpunkt betrug der Durchmesser des Wasserrades 5,68 Meter. ${ }^{50}$ Die Mühle wechselte später dreimal den Besitzer, im Jahre 1928 gehörte sie Károly Toldi, dann Frau János Forrai und im Jahre 1941 Dr. Jenő Ernszt. ${ }^{51}$ Nach einer 1936 entstandenen Beschreibung standen an diesem Wasserlauf elf weitere Mühlen. Aber mit dieser Beschreibung setzte ich mich noch nicht ernsthaft auseinander. ${ }^{52}$

Die Betriebe am Ufer des Tettye-Bachs können aufeine größere Vergangenheitzurückblicken, aber über die getreidemahlenden Mühlen stehen wenige Daten zur Verfügung. Der Ingenieur Ignátz Lukrits vermaß im Jahre 1878 die Besitzungen der ganzen Stadt, darunter auch die Mühlen am Tettye. Damals arbeiteten hier auch eine Sägemühle und eine Schießpulvermühle. Eine explodierte im Jahre 1832.„Die Wasserräder waren meistens oberschlächtig, ein unterschlächtiges Wasserrad hatten nur zwei Müblen (eine mit einem Rad und eine mit zwei Räder). Der Tettye-Bach ist tief, so konnte hier nicht nur eine große Zabl an Müblen gebaut werden, sondern auch Mühlräder mit großem Durchmesser. Die Durchmesser der Räder betrug zwischen drei und vier Metern. 16 Müblen hatten Räder mit einem Durchmesser zwischen vier und fünfMeter, 18 Müblen hatten Räder

\footnotetext{
Ders. 24.

Ders. 25

Ders. 27-28.

Ders. 30.

Ders. 32.

Ders.

Ders.
} 
größer als fünf Meter 12 Stück (...) (Ausnahme von mir R.B.ZS.) Es arbeiteten also 30 Müblen mit insgesamt 48 Rädern, davon war 3 unterschlächtig. " 53

Zusammenfassend kann man sagen, dass die Müller aus dem Komitat Baranya an dem ländlichen Müllerleben teilnahmen. Sie versuchten ihre Interesse sowohl auf der lokalen als auch auf der regionalen Ebene durchzusetzen. Die Mühlenbesitzer in dem Komitat Baranya waren so organisiert, wie die Mühlenbesitzer der Epoche in anderen Komitaten. Die Fünfkirchner Kleinmühlen arbeiteten an den vorher erwähnten Wasserläufen. Oft wechselten die Mühlen Besitzer und die Durchmesser der Räder wurden vergrößert, um mit mehr Kraft antreiben zu können. An den meisten Orten gab es früher schon Mühlen. Ihr Schicksal hing oft davon ab, welcheInteressen die Donaudampfschiff-Gesellschaft mit den Gewässern hatte.Die Gesellschaft besitzten Kohlenbergwerke, die an mehreren Orten die Wasserläufe mehr oder weniger beseitigten, so dass diese Wasserläufe keine Mühlräder mehr antreiben konnten. Das Schicksal der Mühlen war unterschiedlich, aber man kann sagen, dass sie mit ähnlichen Schwierigkeiten zu kämpfen hatten. In mehreren Fällen kam es vor, dass erst nach ein oder zwei Jahrzehnten nachdem die Mühle das Mahlen eingestellt hatte, die Stadt das Wassernutzungsrecht widerrief. In der Zwischenzeit konnten die Mühlen schon nicht mehr mahlen, meistens deswegen, weil die natürlichen Bedingungen dies nicht mehr erlaubten. Die historischen Ereignisse, Krisen und Verstaatlichungsgesetze besiegelten ihr Schicksal schließlich endgültig. ${ }^{54}$ 